\title{
Satisfação e responsividade em serviços de atenção à saúde da Fundação Oswaldo Cruz
}

\author{
User satisfaction and responsiveness in the healthcare services \\ at Fundação Oswaldo Cruz
}

Eliane Hollanda ${ }^{1}$

Sandra Aparecida Venâncio de Siqueira ${ }^{1}$

Gabriela Rieveres Borges de Andrade ${ }^{1}$

Alex Molinaro

Jeni Vaitsman ${ }^{1}$

${ }^{1}$ Departamento de Ciências Sociais, Escola Nacional de Saúde Pública (Fiocruz). Rua Leopoldo Bulhões 1.480/906, Manguinhos. 21041-210 Rio de Janeiro RJ.elianeh@ensp.fiocruz.br

\begin{abstract}
The paper discusses the results of research into user satisfaction in three healthcare facilities at Oswaldo Cruz Foundation in Rio de Janeiro. The analysis is based on the concepts of user satisfaction and responsiveness. Perceptions and opinions of outpatients from Instituto de Pesquisa Evandro Chagas, Instituto Fernandes Figueira and Centro de Saúde Escola Germano Sinval Faria were investigated. Intention samples were drawn for each institution and a total of 1.339 valid questionnaires were obtained. The study found that patients are satisfied with healthcare at Fiocruz, especially with health professionals. Nevertheless, restroom hygiene and privacy during consultations were less well evaluated. The improvement of these aspects depends not only on financial and technological investments but on changes in organizational culture. The first part of the paper discusses the literature on user satisfaction and responsiveness, with the definition of the categories that guided the study. The methodology is then presented as well as the four health services dimensions assessed - dignity, agility, facilities and communication - and the results are analyzed.
\end{abstract}

Key words User satisfaction, Responsiveness, Evaluation, Out-patient clinics, Survey
Resumo O artigo discute resultados de uma pesquisa sobre satisfação dos usuários com o atendimento em três unidades assistenciais da Fundação Oswaldo Cruz na Cidade do Rio de Janeiro, tomando como base as categorias satisfação do usuário e responsividade. Pesquisou-se as percepções e as opiniões de pacientes ambulatoriais sobre o atendimento no Instituto de Pesquisa Clínica Evandro Chagas, Instituto Fernandes Figueira e Centro de Saúde Escola Germano Sinval Faria. Foram aplicados questionários a partir de amostras intencionais para cada unidade, em um total de 1.339 questionários válidos. A pesquisa encontrou uma alta satisfação entre os usuários, principalmente com o atendimento dos profissionais de saúde. Porém, itens específicos, tais como limpeza dos banheiros e privacidade durante as consultas, não foram bem avaliados. A melhoria desses aspectos depende não somente de investimentos em recursos financeiros e tecnológicos, mas de mudanças na cultura organizacional. Inicialmente é feita uma discussão da literatura sobre satisfação do usuário, identificando os conceitos que nortearam a pesquisa. Em segui$\mathrm{da}$, descrevem-se as etapas metodológicas, as quatro dimensões dos serviços avaliadas - dignidade, agilidade, instalações e informação - e se analisa os resultados da pesquisa.

Palavras-chave Satisfação do usuário, Responsividade, Atendimento, Ambulatório, Survey 


\section{Introdução}

$\mathrm{O}$ artigo discute resultados de uma pesquisa sobre satisfação dos usuários com o atendimento em três unidades assistenciais da Fundação Oswaldo Cruz (Fiocruz) na cidade do Rio de Janeiro: Instituto Fernandes Figueira (IFF), Instituto de Pesquisa Clínica Evandro Chagas (IPEC) e Centro de Saúde Escola Germano Sinval Faria/ Escola Nacional de Saúde Pública (CSEGSF/ ENSP). O texto apresenta uma síntese dos resultados obtidos em unidades com níveis de atenção e clientela diferenciadas.

Tais diferenças colocaram o desafio de elaborar o desenho de pesquisa de forma que sua aplicação aos três contextos não desconsiderasse as especificidades de cada um e necessidade de interpretar os resultados considerando as variações em tais contextos.

Para analisar o grau de satisfação dos usuários da Fiocruz com os serviços prestados tomou-se como base a tendência observada na literatura que descreve variação inversa entre grau de expectativa e grau de satisfação. A hipótese deste estudo é de que os usuários seriam mais exigentes na análise dos serviços prestados por esta instituição, apresentando, portanto, satisfação mais baixa, em razão da alta expectativa que teriam com o atendimento ofertado, motivados pelo fato da Fiocruz ser considerada uma ilha de excelência no setor saúde e gozar de grande prestígio junto à população.

A pesquisa foi solicitada pela Ouvidoria da Fiocruz a uma equipe de trabalho do departamento de Ciências Sociais da ENSP com experiência acumulada no tratamento do tema Satisfação do Usuário ${ }^{1}$. A Ouvidoria, criada em 2004, examina e encaminha às instâncias competentes as manifestações dos usuários, propondo ações que contribuam para o desenvolvimento dos padrões de desempenho da instituição e a elevação do grau de satisfação de seus usuários. Como decorrência destas atividades, oferece subsídios aos gestores para a modificação das práticas assistenciais e pretende favorecer o aprendizado institucional. A solicitação desta pesquisa decorreu do pressuposto de que uma das funções da Ouvidoria é estimular avaliações sistemáticas sobre a satisfação dos usuários da Fiocruz. Por seu caráter aplicado, estudos de satisfação de usuários permitem que o conhecimento produzido subsidie a construção de tecnologias de monitoramento e avaliação. $\mathrm{O}$ trabalho foi financiado parcialmente pela Ouvidoria, que concedeu bolsas para contratação de dois pesquisa- dores e dos estagiários que desenvolveram o trabalho de campo.

$\mathrm{O}$ artigo faz inicialmente uma discussão da literatura sobre satisfação do usuário, identificando os conceitos básicos que nortearam a pesquisa. Em seguida, descreve as etapas metodológicas e as quatro dimensões dos serviços avaliadas - dignidade, agilidade, instalações e informação - e analisa os resultados da pesquisa.

\section{Satisfação do usuário: contexto e conceito}

As pesquisas de satisfação na área da saúde começam a ser feitas na década de 1970, visando a adesão dos pacientes ao tratamento médico ${ }^{2}$. Com Donabedian ${ }^{3}$, tornam-se um instrumento para medir também a qualidade dos serviços. Diferentes modelos orientam estas pesquisas, levando em conta diferentes dimensões e componentes da satisfação ${ }^{4}$.

A avaliação dos serviços pelos usuários popularizou-se na Europa e nos EUA a partir dos anos 1980, em geral através de surveys. No setor saúde, a concepção de administração pública voltada para a qualidade deu aos pacientes um lugar importante na avaliação dos serviços. A satisfação do usuário começou a ser vista como legítima, tornando-se um atributo da ideia de qualidade, um objetivo em si e não apenas um meio de fazer com que o paciente aderisse ao tratamento, como era comum nos objetivos de estudos anteriores.

No Brasil, a difusão desses inquéritos se deu no contexto das várias tentativas de reformas e melhoria da administração pública que, a partir da década de 1990, começaram a incorporar o conceito de qualidade como controle dos resultados em vez do controle de meios, como no modelo de administração burocrático ${ }^{5-8}$.

Os trabalhos de revisão da literatura sublinham a inexistência de consenso sobre o que venha a ser satisfação do usuário; parte do problema reside em conceituar os termos "satisfação" e "usuário"; outro aspecto é a multiplicidade de abordagens teóricas sobre a satisfação, tidas como frágeis ou pouco desenvolvidas ${ }^{4,9}$. Observa-se que as pesquisas medem a satisfação de "pacientes", "usuários" ou "consumidores", dependendo do modelo utilizado; mas há concordância em que todos se baseiam nas percepções do paciente em relação às suas expectativas, valores e desejos ${ }^{10,11}$.

A ideia de satisfação do usuário define o paciente como sujeito e coloca suas opiniões, expectativas e tudo o que ele considera justo ou equâni- 
me como elementos legítimos na definição e na mensuração da qualidade da atenção à saúde. Expressa, neste sentido, uma atitude, uma resposta afetiva com base na crença de que o cuidado possui certos atributos que os indivíduos têm condições de avaliar. A satisfação, neste contexto, é vista como uma atitude positiva do usuário ${ }^{12}$.

No âmbito do SUS, pesquisas de satisfação do usuário ganharam destaque a partir de iniciativas voltadas para o fortalecimento da cultura participativa, dos direitos do paciente e do atendimento voltado para as suas necessidades ${ }^{13}$. Uma dessas iniciativas é o Programa Nacional de Humanização do Atendimento que valoriza, entre outras coisas, a qualidade da interação dos profissionais de saúde com os pacientes ${ }^{14}$.

A popularidade das pesquisas com foco na opinião do usuário de serviços públicos e, em especial, de serviços de saúde, não exclui críticas. As avaliações positivas obtidas pela maioria das pesquisas de satisfação sobre o atendimento em saúde em países em desenvolvimento têm sido questionadas por vários autores ${ }^{15,16}$. Chama atenção o fato de que essas pesquisas partem do pressuposto de que todos os usuários desempenhem um papel crítico e a possibilidade da aceitação passiva do serviço não é considerada. Autores apontam, no entanto, a existência do viés da gratidão, sentimento que certos pacientes podem apresentar quando conseguem atendimento, fazendo com que possíveis problemas ligados ao atendimento fiquem em segundo plano ${ }^{16}$.

Outro problema das pesquisas de satisfação do usuário é a diversidade de metodologias e instrumentos de pesquisa, o que dificulta a comparabilidade entre elas. Além disso, essas pesquisas tendem a privilegiar a expectativa do respondente em detrimento da avaliação propriamente dita dos serviços de saúde ${ }^{17}$. Tanto a satisfação quanto as expectativas podem variar de acordo com variáveis como gênero, idade, classe social, escolaridade, entre outras ${ }^{18,19}$. Uma baixa expectativa em relação ao serviço tende a resultar em maior satisfação bem como um alto grau de exigência tende a levar a uma menor satisfação com o serviço, visto ser mais difícil corresponder a uma alta expectativa ${ }^{12,16}$.

Vendo a questão da satisfação por outro ângulo, a Organização Mundial de Saúde ${ }^{20}$ propôs o conceito de responsividade para avaliar a performance dos sistemas nacionais de saúde. A responsividade (responsiveness) seria um dos objetivos intrínsecos do sistema de saúde e refere-se à quão bem as expectativas legítimas da população estão sendo atendidas. São expectativas legí- timas os elementos não diretamente ligados ao estado de saúde, mas que afetam a relação do usuário com os serviços e com os profissionais, tais como atendimento digno e respeitoso, respeito à confidencialidade das informações e à privacidade durante as consultas, autonomia para escolher entre opções de tratamento ou profissionais, atendimento ágil com o menor tempo de espera possível e oferta de apoio social.

Para mensurar esses aspectos, as pesquisas sobre responsividade fazem perguntas sobre $o$ que acontece quando o usuário interage com o sistema ao invés de questionar sobre a sua satisfação. Com isso, procura minimizar o aspecto subjetivo dessas pesquisas, pois avalia as percepções individuais sobre o sistema de saúde em relação às "expectativas legitimadas universalmente”. Assim, independentemente das expectativas das pessoas, existem parâmetros definidos como legítimos e universais pela Organização Mundial de Saúde para avaliar os serviços de saúde ${ }^{21}$.

A responsividade privilegia a avaliação de aspectos não médicos do atendimento, ou seja, aqueles não diretamente ligados ao estado de saúde, mas que influem na saúde dos pacientes e que são mais facilmente observados e julgados por estes ${ }^{11}$. Neste ponto, se diferencia das pesquisas de satisfação que tendem a incorporar dimensões técnicas, tais como competência dos profissionais e efetividade ${ }^{22,23}$. Esta pesquisa optou por privilegiar dimensões não médicas do atendimento.

\section{Metodologia da pesquisa}

\section{Locais da pesquisa}

O IPEC, criado em 1918, é especializado no estudo de doenças infecciosas, considerado centro de referência de nesta área. $\mathrm{O}$ atendimento é feito por médicos especializados em doenças infecciosas e integrado aos serviços clínicos complementares (enfermagem, nutrição, farmácia, psicologia, serviço social e fisioterapia). Os programas de atendimento vinculam-se às linhas de pesquisa do hospital e, conforme a indicação clínica, o paciente pode ser encaminhado para ambulatório, hospital-dia ou internação.

O IFF, criado em 1924, presta atendimento em Saúde da Mulher, da Criança e do Adolescente. Recebe pacientes gestantes de alto risco referenciados pela rede pública de atendimento do município e do Estado do Rio de Janeiro. Possui Ambulatório Geral (Pré-Natal, Ginecologia, Pediatria e 
outros) e Especializado (Neurologia, Pneumologia, Endopediatria, Neurocirurgia e outros).

O CSEGSF/ENSP foi criado em 1967 com a finalidade de atender prioritariamente a população moradora no Complexo de Manguinhos, no município de Rio de Janeiro, onde está localizado, e ser campo de ensino e pesquisa em atenção primária para ENSP/Fiocruz. Contava, no momento da pesquisa, com quinze consultórios para atendimento individual multidisciplinar, três salas para grupos e infraestrutura de serviços complementares, incluindo trabalhos de educação, prevenção e promoção da saúde dentro da comunidade.

\section{Dimensões avaliadas}

As dimensões do atendimento avaliadas nesta pesquisa foram definidas com base na revisão bibliográfica e em pesquisa anterior realizada no IPEC entre 2000 e $2001^{1,22}$. Essas dimensões são tradicionais em pesquisas sobre satisfação dos usuários, além de englobar outras, derivadas do conceito de Responsividade 20,21 .

$\mathrm{O}$ estudo concentrou-se em quatro (4) dimensões relativas ao atendimento: Agilidade, Dignidade, Instalações e Informações. Embora a pesquisa tenha explorado diferentes aspectos relativos a cada dimensão, este artigo focaliza os aspectos centrais de cada uma.

Sobre a dimensão Agilidade, são apresentados os resultados relativos ao tempo de espera para a consulta e o tempo de espera que o paciente considera tolerável esperar. Em relação à Dignidade, são focalizados o atendimento respeitoso dos profissionais de saúde, a confiança na confidencialidade das informações do prontuário e a privacidade durante consultas e exames. Sobre a dimensão Instalações, o artigo mostra a avaliação da limpeza das salas de espera e dos consultórios. Sobre a dimensão Informações, apresenta-se a avaliação da facilidade/dificuldade em conseguir informações na unidade. Além disso, foi solicitado que o paciente desse uma nota de 0 a 10 para expressar a expectativa que tinha em relação à unidade. Solicitou-se, igualmente, uma nota de 0 a 10 para quantificar a satisfação ou insatisfação com cada dimensão e com a unidade bem como com o atendimento na unidade como um todo.

\section{Instrumento de coleta dos dados e trabalho de campo}

O questionário aplicado foi estruturado em oito blocos, sendo o primeiro de verificação dos critérios de inclusão na pesquisa e o último, de caracterização do perfil do entrevistado. Os outros seis blocos incluíam questões sobre aspectos do atendimento a partir das categorias definidas - Agilidade, Dignidade, Instalações e Informações. Ao todo, foram feitas 86 perguntas; 80 com opções de respostas fechadas e $6 \mathrm{com}$ opções de respostas abertas ${ }^{24}$.

O questionário foi pré-testado em cinquenta usuários do CSEGSF/ENSP, para avaliar a facilidade dos aplicadores na leitura das questões, a compreensão das questões pelo respondente, a adequação da linguagem e da ordem das questões. Os ajustes foram feitos de forma que o tempo médio de aplicação do questionário não ultrapassasse 20 minutos.

Alunos de graduação de Medicina foram treinados e entrevistaram, durante um período de três meses, pacientes ambulatoriais em diferentes dias e turnos (manhã/tarde) de semanas típicas (sem a presença de feriado). As entrevistas foram realizadas no local de espera para consultas. O trabalho de campo foi supervisionado pela equipe de pesquisa. Antes do início do trabalho de campo, a pesquisa foi apresentada às equipes de cada unidade que autorizaram e colaboraram com a realização da pesquisa. Os objetivos da pesquisa foram explicitados aos respondentes, através da leitura do Termo de Consentimento Livre e Esclarecido, aprovado pelo Comitê de Ética em Pesquisa da ENSP/Fiocruz.

\section{Amostra}

Foram pesquisadas opiniões e percepções de pacientes ambulatoriais do IFF, do IPEC e do CSEGSF/ENSP e/ou seus familiares, nos casos de crianças, a partir de amostra intencional por cotas. O desenho da amostra considerou a análise de planilhas de atendimento fornecidas pelas unidades, sendo o primeiro critério de seleção a utilização de semanas-padrão de atendimento (sem a presença de feriados). A amostra intencional por cotas, em que inicialmente se descrevem algumas características da população-alvo, mostrou-se o tipo de desenho mais adequado, dada a inexistência de cadastro atualizado de usuários em todas as unidades pesquisadas, o tempo e o custo de uma amostra probabilística.

A partir da elaboração da amostra, foram aplicados 447 questionários no CSEGSF/ENSP, 453 questionários no IFF e 439 questionários no IPEC, totalizando 1.339 questionários válidos.

Para cada unidade, foram utilizados critérios de seleção de entrevistados diferenciados, de acor- 
do com as características do atendimento e/ou do perfil dos usuários. O pressuposto para a alocação da amostra no CSEGSF/ENSP foi a distribuição conjunta por sexo, faixa etária e atendimento, ou não, pelo PSF. Dos 447 pacientes entrevistados, $82 \%$ eram cadastrados no PSF; $18 \%$ receberam cuidados de outros profissionais do CSEGSF/ENSP.

No IPEC, a amostra foi distribuída de forma proporcional entre homens e mulheres e por patologia. No período de março a junho de 2007, cerca de 50\% dos atendimentos realizados dirigiram-se a pacientes com HIV/HTLV e os outros $50 \%$ às demais patologias, sendo mantida essa proporção na amostra selecionada. A proporção de entrevistas realizadas com pacientes de outras patologias também foi controlada A mais frequente, depois de HIV/AIDS, foi a Doença de Chagas, que afetava $15 \%$ dos respondentes.

No IFF, a escolha dos entrevistados foi controlada por tipo de ambulatório, se geral ou especializado, e por tipo de atendimento. As entrevistas realizadas nos ambulatório geral corresponderam a $72 \%$ das entrevistas realizadas no IFF. As entrevistas com pacientes de Ginecologia corresponderam a $29 \%$ daquele total $(n=131)$ e com pacientes de Pediatria, a $30 \%$ do total $(\mathrm{n}=$ 136). Para ambulatório especializado, $18 \%$ das entrevistas $(n=23)$ foram feitas com pacientes de Follow up (acompanhamento), 16,5\% com pacientes de Pneumologia $(\mathrm{n}=21), 15,7 \%$ com pacientes de Neurologia ( $\mathrm{n}=20)$ e $14 \%$ com pacientes de Nutrição $(\mathrm{n}=18)$. As demais entrevistas foram realizadas com pacientes de Alergia ( $\mathrm{n}$ $=11)$, DIP $(\mathrm{n}=9)$, Adolescentes $(\mathrm{n}=7)$, Cardiologia $(n=6)$, Neurocirurgia $(n=5)$ e outros $(n=$ 4), totalizando 127 entrevistas (36\%).

Os dados oriundos dos questionários foram sistematizados em banco de dados no SPSS. A consistência destas variáveis foi verificada através da análise de frequência. Para análise dos dados foram utilizados instrumentais da estatística descritiva e o teste de correlação de Pearson.

\section{Resultados e discussão}

O universo da pesquisa foi composto em sua maioria por pessoas do sexo feminino, $(71 \%)$, com idades entre 20 e 59 anos (76\%), vivendo maritalmente $(46,5 \%)$, com escolaridade fundamental $(68,8 \%)$ e renda média situada entre um e dois salários mínimos. Em relação à cor/raça, $38,8 \%$ se definiram como pardos; $33,3 \%$ como brancos; $25,9 \%$ como negros.
Estudos de satisfação do usuário no Brasil têm mostrado que a escolaridade é uma dimensão que tende a influir na satisfação com o atendimento. Entre os usuários do SUS, os grupos com maior escolaridade tendem a ser mais exigentes com a qualidade do atendimento e, portanto, mostram-se menos satisfeitos ${ }^{18,25}$. Neste estudo, no entanto, não foram verificadas correlações significativas entre variáveis relativas ao perfil do usuário (escolaridade, idade, sexo e renda) e a nota atribuída pelos usuários à satisfação geral com o atendimento, o que pode ser explicado pela homogeneidade verificada nas características sociais deste grupo.

\section{Expectativa e Satisfação}

Um dos aspectos que influenciam o grau de satisfação dos usuários com o serviço de saúde está relacionado à expectativa destes indivíduos com a qualidade do serviço no qual serão atendidos. Por sua vez, um dos elementos que moldam a expectativa é a experiência prévia com outras unidades de saúde.

Para confirmar a hipótese deste estudo de que os usuários apresentariam menor grau de satisfação com o atendimento prestado pela Fiocruz em decorrência da alta expectativa com a qualidade destes foi necessário, inicialmente, identificar se na avaliação dos usuários os serviços prestados por esta instituição eram melhores do que os ofertados por outros serviços da rede pública. Para tanto, foi solicitado que respondessem se na avaliação deles a unidade da Fiocruz que frequentam é "muito melhor", "melhor", "igual", "pior" ou "muito pior" do que outras unidades públicas de saúde que conhece.

A maioria (76\%) considera os serviços prestados na unidade da Fiocruz que frequenta "muito melhor" ou "melhor" do que os de outras unidades de saúde. Outros $10 \%$ avaliam as unidades como sendo iguais às demais e $6 \%$ responderam "pior" ou "muito pior" do que as outras. $\mathrm{O}$ restante $(8 \%)$, não soube responder.

A média das notas atribuídas à satisfação com o atendimento prestado nas unidades foi de 9,1 (Tabela 1), ou seja, mais alta do que a média das notas para a expectativa $(8,4)$. Além disso, não foi encontrada correlação (Tabela 2) entre satisfação e expectativa $(\mathrm{P}=0,141)$. Estes resultados invibilizam a aceitação da hipótese da existência de uma correlação inversa entre satisfação e expectativa.

A média das notas para a satisfação, embora alta para todas as unidades pesquisadas, foi menor no CSEGSF/ENSP (Tabela 1). Essa diferença 
pode, em parte, ser explicada pelo grau de especialização dos serviços ofertados pelo IPEC e pelo IFF. Em geral, serviços mais especializados tendem a ser mais bem avaliados devido à sua exclusividade (poucos tem acesso a tais serviços) e ao acesso mais fácil a exames e outros procedimentos complexos.

Nas notas atribuídas às dimensões do atendimento, o desempenho dos profissionais de saúde foi melhor avaliado pelos usuários das unidades da Fiocruz. Outros estudos também encontraram essa valorização dos profissionais pelos pacientes que tendem a avaliar sua competência através de atributos tais como gentileza, respeito, compreensão ${ }^{26,27}$.

O tempo de espera para consultas foi o aspecto com o qual os usuários da Fiocruz ficaram menos satisfeitos, tendo este resultado se repetido nas três unidades. Outros estudos têm mostrado que o tempo de espera para consultas é um alvo recorrente de insatisfação ${ }^{18,28}$.

Os dados da Tabela 2 indicam que a dimensão que apresentou maior correlação com as notas atribuídas à satisfação geral foi atendimento

Tabela 1. Média das notas atribuídas à expectativa e à satisfação com o atendimento geral e com dimensões do atendimento prestado nas unidades da FIOCRUZ - IPEC, IFF, CSEGSF/ENSP.

\begin{tabular}{|c|c|c|c|c|c|c|c|c|}
\hline & \multicolumn{2}{|c|}{ FIOCRUZ } & \multicolumn{2}{|c|}{ IPEC } & \multicolumn{2}{|c|}{ IFF } & \multicolumn{2}{|c|}{ CSEGSF/ENSP } \\
\hline & Média & $\mathbf{N}$ & Média & $\mathbf{N}$ & Média & $\mathbf{N}$ & Média & $\mathbf{N}$ \\
\hline Expectativa & 8,4 & 1195 & 8,5 & 403 & 8,4 & 426 & 8,3 & 366 \\
\hline Satisfação Geral & 9,1 & 1336 & 9,5 & 439 & 9,2 & 453 & 8,6 & 444 \\
\hline Atendimento dos profissionais & 9,0 & 1338 & 9,6 & 439 & 9,2 & 453 & 8,3 & 446 \\
\hline Limpeza & 8,4 & 1339 & 8,8 & 439 & 8,2 & 453 & 8,0 & 446 \\
\hline Facilidade para conseguir informações & 8,4 & 1324 & 9,3 & 435 & 8,4 & 449 & 7,4 & 440 \\
\hline Tempo de espera & 6,8 & 1338 & 7,9 & 439 & 6,6 & 452 & 5,9 & 446 \\
\hline
\end{tabular}

Fonte: Pesquisa satisfação dos usuários em três unidades de atenção à saúde da Fundação Oswaldo Cruz, 2008.

Tabela 2. Correlação de Pearson entre as notas atribuídas à satisfação geral com a qualidade do atendimento e as notas atribuídas à satisfação com a limpeza, o atendimento dos profissionais, o tempo de espera para atendimento e a facilidade para conseguir informações.

\begin{tabular}{|c|c|c|c|c|c|}
\hline $\begin{array}{c}\text { Aspectos } \\
\text { correlacionados }\end{array}$ & Limpeza & $\begin{array}{l}\text { Atendimento } \\
\text { dos } \\
\text { profissionais }\end{array}$ & $\begin{array}{l}\text { Tempo de } \\
\text { espera }\end{array}$ & $\begin{array}{c}\text { Facilidade para } \\
\text { conseguir } \\
\text { informações }\end{array}$ & $\begin{array}{c}\text { Satisfação } \\
\text { Geral }\end{array}$ \\
\hline \multicolumn{6}{|l|}{ Limpeza } \\
\hline $\mathrm{P}$ & 1 & $0,500^{* *}$ & $0,434^{* *}$ & $0,424^{* *}$ & $0,328^{* *}$ \\
\hline $\mathrm{N}$ & 1324 & 1324 & 1324 & 1324 & 1322 \\
\hline \multicolumn{6}{|c|}{ Atendimento dos profissionais } \\
\hline $\mathrm{P}$ & $0,500^{* *}$ & 1 &, $489^{* *}$ &, $526^{* *}$ &, $509^{* *}$ \\
\hline $\mathrm{N}$ & 1324 & 1324 & 1324 & 1324 & 1322 \\
\hline \multicolumn{6}{|l|}{ Tempo de espera } \\
\hline $\mathrm{P}$ &, $434^{* *}$ &, $489^{* *}$ & 1 &, $487^{* *}$ &, $407^{* *}$ \\
\hline $\mathrm{N}$ & 1324 & 1324 & 1324 & 1324 & 1322 \\
\hline \multicolumn{6}{|l|}{$\begin{array}{l}\text { Facilidade para conseguir } \\
\text { informações }\end{array}$} \\
\hline $\mathrm{P}$ &, $424^{* *}$ &, $526^{* *}$ &, $487^{* *}$ & 1 &, $368^{* *}$ \\
\hline $\mathrm{N}$ & 1324 & 1324 & 1324 & 1324 & 1322 \\
\hline \multicolumn{6}{|l|}{ Satisfação Geral } \\
\hline $\mathrm{P}$ &, $328^{* *}$ &, $509^{* *}$ &, $407^{* *}$ &, $368^{* *}$ & 1 \\
\hline $\mathrm{N}$ & 1322 & 1322 & 1322 & 1322 & 1322 \\
\hline
\end{tabular}

** correlação significativa a 0,01

Fonte: Pesquisa satisfação dos usuários em três unidades de atenção à saúde da Fundação Oswaldo Cruz, 2008. 
prestado pelos profissionais $(\mathrm{P}=0,509)$. Estes dados sugerem que os indivíduos que se mostraram satisfeitos com a qualidade geral do atendimento também se mostraram satisfeitos com o atendimento dos profissionais. A limpeza foi a variável que apresentou a menor correlação com a satisfação geral, o que indica que este aspecto influenciou pouco a avaliação dos usuários sobre a qualidade geral do atendimento na unidade.

Embora a correlação entre a dimensão tempo de espera e a satisfação com atendimento ( $P$ $=0,407)$ não seja tão evidente, como o verificado em outras dimensões, não pode ser desconsiderada. Outros achados da pesquisa mostram que a demora em ser atendido é um motivo de reclamação entre os usuários, em decorrência de aguardarem mais tempo (90 $\mathrm{min}$ ) do que consideram tolerável esperar o atendimento (60 min).

\section{Responsividade e Satisfação}

Neste artigo, considerou-se igualmente, como "satisfação" dos usuários com os serviços de saúde prestados, os aspectos do atendimento que são parte da categoria responsividade, ou seja, agilidade, dignidade, instalações e informações (Tabela 3).

Em relação à agilidade do atendimento, uma das dimensões avaliadas foi o tempo que o usuário esperou para ser atendido no dia da entrevista. Em outra pergunta do questionário, os entrevistados disseram achar tolerável esperar até uma hora para o atendimento. Tomando esse dado como parâmetro para a satisfação com o tempo de espera, verificou-se que, em média $40 \%$ dos entrevistados da Fiocruz esperaram acima deste tempo para serem atendidos, o que explica sua insatisfação com este aspecto. No IPEC este percentual foi menor $(28,5 \%)$ do que o verificado nas demais unidades. Estes achados corroboram os resultados encontrados em pesquisa sobre satisfação de pacientes na Pesquisa Mundial de Saúde, realizada no Brasil em $2003^{19}$.

$\mathrm{O}$ atendimento prestado pelos profissionais da Fiocruz foi, no geral, muito bem avaliado pelos usuários. Apesar disto, foram encontradas variações nos valores entre as unidades, as categorias profissionais e os setores analisados. Numa perspectiva de melhoria da qualidade dos serviços e treinamento de mão de obra estas diferenças devem ser levadas em consideração.

Aproximadamente 68\% dos usuários da Fiocruz demonstraram muita confiança nas unidades para assegurar o sigilo das informações contidas no prontuário clínico. No entanto, cerca de
10\% destes usuários informaram ter pouca ou nenhuma confiança na instituição para guardar seus dados de modo seguro, sendo este percentual maior no CSEGSF (14\%). Este aspecto, em tese, poderia ter sido mais bem avaliado pelo usuário e objeto de seu questionamento junto às unidades. No entanto, na literatura, este aspecto não parece ser muito considerado na avaliação dos usuários. Pesquisa da Organização Mundial de Saúde para 41 países mostra que a confidencialidade aparece apenas em $5^{\circ}$ lugar entre as dimensões consideradas mais importantes pelos usuários na avaliação dos cuidados em saúde ${ }^{29}$.

A privacidade nas consultas foi o aspecto da responsividade que os usuários identificaram maior falha nos serviços. Cerca de $60 \%$ dos entrevistados afirmaram que, durante a realização da consulta ou exame, outros profissionais entraram na sala sem solicitar permissão. No IPEC, este percentual foi maior do que o verificado nas demais unidades $(65,8 \%)$. A inexistência de local apropriado para a troca de roupa para a realização de exames foi outro aspecto da privacidade apontado como deficitário pela maioria $(56,4 \%)$ dos usuários da Fiocruz, o que aponta para a necessidade de readequação dos espaços onde funcionam estas unidades.

Cerca de $80 \%$ dos entrevistados consideraram a qualidade da limpeza das instalações "boa" ou "muito boa". No entanto, a limpeza dos banheiros apresenta um percentual de satisfação bem abaixo desta média (58\%), à exceção do CSEGSF (73\%). O fato de esta unidade encontrar-se em obras, durante o período de aplicação do questionário, pode ter aumentado a tolerância dos usuários.

Por fim, outro aspecto importante da responsividade é a facilidade que o usuário encontra nas unidades de saúde para conseguir as informações necessárias sobre o atendimento dos profissionais, locais de exames, consultas e horários de atendimento. A maioria dos entrevistados considerou “fácil” ou "muito fácil” conseguir informações nas unidades da Fiocruz.

\section{Considerações Finais}

Os dados apresentados mostram que, embora a satisfação dos usuários da Fiocruz com o atendimento prestado pelas unidades seja alta, existe variação entre as unidades em relação a qual aspecto do atendimento os usuários estão mais ou menos satisfeitos. Um fator que contribui para esta diferenciação é o tipo de atendimento pres- 
Tabela 3. Responsividade em unidades assistenciais da Fiocruz - CSEGSF, IFF, IPEC.

\begin{tabular}{|c|c|c|c|c|}
\hline Categorias & FIOCRUZ & CSEGSF & IFF & IPEC \\
\hline \multicolumn{5}{|l|}{ a)AGILIDADE } \\
\hline \multicolumn{5}{|l|}{ Tempo de espera para ser atendido } \\
\hline Até 30 minutos & $32,9 \%$ & $33,3 \%$ & $22,7 \%$ & $42,8 \%$ \\
\hline 30 a 60 minutos & $25,2 \%$ & $22,4 \%$ & $25,6 \%$ & $27,6 \%$ \\
\hline 60 a 120 minutos & $20,6 \%$ & $17,4 \%$ & $24,7 \%$ & $19,6 \%$ \\
\hline Mais de duas horas & $18,5 \%$ & $23,7 \%$ & 22,70 & $8,9 \%$ \\
\hline \multicolumn{5}{|l|}{ b)DIGNIDADE } \\
\hline \multicolumn{5}{|l|}{ Atendimento respeitoso por: } \\
\hline Médico & $95,0 \%$ & $91,9 \%$ & $96,0 \%$ & $98,4 \%$ \\
\hline Enfermagem & $90,6 \%$ & $86,1 \%$ & $90,7 \%$ & $95,0 \%$ \\
\hline Triagem & $90,6 \%$ & $83,4 \%$ & $88,1 \%$ & $88,1 \%$ \\
\hline Recepção & $89,9 \%$ & $85,9 \%$ & $89,4 \%$ & $94,8 \%$ \\
\hline Laboratório & $89,6 \%$ & $87,5 \%$ & $86,0 \%$ & $95,7 \%$ \\
\hline Farmácia & $90,2 \%$ & $92,4 \%$ & $85,4 \%$ & $92,9 \%$ \\
\hline Segurança & $88,7 \%$ & $84,8 \%$ & $93,2 \%$ & $88,1 \%$ \\
\hline Limpeza & $88,0 \%$ & $83,0 \%$ & $92,0 \%$ & $88,8 \%$ \\
\hline \multicolumn{5}{|l|}{ Confidencialidade das informações do prontuário } \\
\hline Muita confiança & $68,3 \%$ & $65,8 \%$ & $63,8 \%$ & $75,4 \%$ \\
\hline Alguma confiança & $17,4 \%$ & $15,0 \%$ & $21,2 \%$ & $15,9 \%$ \\
\hline Pouca confiança & $7,7 \%$ & $10,7 \%$ & $8,6 \%$ & $3,6 \%$ \\
\hline Nenhuma confiança & $2,4 \%$ & $3,4 \%$ & $3,3 \%$ & $0,5 \%$ \\
\hline \multicolumn{5}{|l|}{ Privacidade } \\
\hline Existência de privacidade na consulta & $40,4 \%$ & $47,6 \%$ & $38,4 \%$ & $34,2 \%$ \\
\hline $\begin{array}{l}\text { Existência de local apropriado para a troca de } \\
\text { roupa para exame físico }\end{array}$ & $40,7 \%$ & $49,9 \%$ & $53,7 \%$ & $20,2 \%$ \\
\hline \multicolumn{5}{|l|}{ c) INSTALAÇÕES } \\
\hline \multicolumn{5}{|l|}{ Limpeza dos locais de espera } \\
\hline Muito Boa & $19,5 \%$ & $16,8 \%$ & $16,1 \%$ & $25,7 \%$ \\
\hline Boa & $62,7 \%$ & $60,2 \%$ & $64,7 \%$ & $63,1 \%$ \\
\hline Regular & $16,8 \%$ & $21,7 \%$ & $17,9 \%$ & $10,7 \%$ \\
\hline Ruim & $0,8 \%$ & $1,1 \%$ & $0,9 \%$ & $0,5 \%$ \\
\hline Não sabe/não respondeu & $0,2 \%$ & $0,2 \%$ & $0,4 \%$ & $0,0 \%$ \\
\hline \multicolumn{5}{|l|}{ Limpeza dos consultórios } \\
\hline Muito Boa & $26,4 \%$ & $21,3 \%$ & $23,0 \%$ & $35,1 \%$ \\
\hline Boa & $67,0 \%$ & $68,9 \%$ & $70,4 \%$ & $61,5 \%$ \\
\hline Regular & $5,8 \%$ & $8,3 \%$ & $6,2 \%$ & $3,0 \%$ \\
\hline Ruim & $0,2 \%$ & $0,7 \%$ & $0,0 \%$ & $0,0 \%$ \\
\hline Não sabe/não respondeu & $0,6 \%$ & $0,9 \%$ & $0,4 \%$ & $0,5 \%$ \\
\hline \multicolumn{5}{|l|}{ Limpeza de Locais de Coleta } \\
\hline Muito Boa & $29,3 \%$ & $27,7 \%$ & $21,2 \%$ & $39,2 \%$ \\
\hline Boa & $55,7 \%$ & $54,1 \%$ & $60,0 \%$ & $52,8 \%$ \\
\hline Regular & $3,1 \%$ & $3,1 \%$ & $3,1 \%$ & $3,2 \%$ \\
\hline Ruim & $0,4 \%$ & $0,7 \%$ & $0,4 \%$ & $0,0 \%$ \\
\hline Não sabe/não respondeu & $11,5 \%$ & $14,3 \%$ & $15,2 \%$ & $4,8 \%$ \\
\hline \multicolumn{5}{|l|}{ Limpeza dos banheiros } \\
\hline Muito Boa & $16,5 \%$ & $22,1 \%$ & $9,7 \%$ & $17,8 \%$ \\
\hline Boa & $41,7 \%$ & $50,8 \%$ & $37,5 \%$ & $36,9 \%$ \\
\hline Regular & $25,5 \%$ & $11,6 \%$ & $35,1 \%$ & $29,6 \%$ \\
\hline Ruim & $9,9 \%$ & $5,1 \%$ & $15,7 \%$ & $8,9 \%$ \\
\hline Não sabe/não respondeu & $6,3 \%$ & $10,3 \%$ & $2,0 \%$ & $6,8 \%$ \\
\hline \multicolumn{5}{|l|}{ D)INFORMAÇÕES } \\
\hline \multicolumn{5}{|l|}{ Facilidade/dificuldade para conseguir informação } \\
\hline Muito difícil & $8,4 \%$ & $8,3 \%$ & $10,1 \%$ & $6,2 \%$ \\
\hline Difícil & $9,5 \%$ & $11,0 \%$ & $10,9 \%$ & $3,7 \%$ \\
\hline Fácil & $58,3 \%$ & $56,9 \%$ & $60,5 \%$ & $58,0 \%$ \\
\hline Muito fácil & $21,7 \%$ & $22,1 \%$ & $17,1 \%$ & $28,4 \%$ \\
\hline Não sabe/ não respondeu & $2,0 \%$ & $1,7 \%$ & $1,6 \%$ & $3,7 \%$ \\
\hline
\end{tabular}

Fonte: Pesquisa satisfação dos usuários em três unidades de atenção à saúde da Fundação Oswaldo Cruz, 2008. 
tado, sendo que as unidades mais especializadas foram mais bem avaliadas. Revelam ainda que, embora residual, persiste certo grau de insatisfação. Esta recaiu na limpeza dos banheiros, na questão da privacidade e na confidencialidade das informações dos prontuários, aspectos que podem ser melhorados nas unidades.

Não se observou, no entanto, como sugere a literatura, correlação entre o perfil dos usuários e as notas atribuídas à expectativa e a satisfação, o que levanta outras hipóteses para explicar a permanência desta insatisfação, que precisariam ser investigadas. Esta tanto pode ser consequência de ocorrências espaçadas, diluídas entre os diferentes grupos de pacientes, quanto localizadas em pacientes que não estão sendo atendidos.

Paralelamente, alguns aspectos relativos à responsividade mostraram-se importantes para explicar o alto grau de satisfação apresentada pelos usuários e para qualificar as perguntas que solicitavam respostas em forma de notas.

A melhoria dos aspectos que não foram bem avaliados (limpeza, tempo de espera e privacidade nas consultas e exames) não dependeria, em princípio, de grandes investimentos em recursos financeiros ou tecnológicos. Remetem ao âmbito da cultura e da gestão organizacional, sobretudo no contexto onde o usuário é a clientela de baixa renda atendida pelo SUS. Deve-se se indagar sobre o lugar simbólico e social dessa população nos serviços públicos onde em geral aspectos relacionados à satisfação talvez não adquiram prioridade, ao contrário dos espaços em que predominam clientelas de renda mais alta. Vê-se, no entanto, que são dimensões do atendimento que fazem parte das expectativas dos usuários.

Além de aspectos intrínsecos à organização dos serviços, a responsividade e a satisfação dos usuários estão relacionados a problemas externos que afetam o desempenho destas unidades: relação com instâncias municipais, estaduais e federais da área da saúde, que limitam o acesso a insumos e disponibilidades de recursos humanos.

Os problemas encontrados neste estudo são comuns a diversas outras organizações de saúde no âmbito do SUS. A Política de Humanização do Ministério da Saúde está sendo desenvolvida no sentido de preservar o acesso e a qualidade de vida dos usuários do sistema, enfatizando aspectos responsivos do cuidado à saúde.

Ao mesmo tempo, as especificidades de cada unidade em relação à clientela, nível de complexidade e missão institucional, impõem desafios organizacionais diferenciados para melhorar os aspectos apontados pela pesquisa. Os itens da pesquisa poderiam ser refinados no futuro, no sentido de incorporar essas especificidades.

Para concluir, ressaltamos que no âmbito das relações entre serviços e usuários, as pesquisas sobre satisfação podem contribuir com a melhoria da qualidade ao mostrar em que medida os problemas afetam o atendimento. Uma vez que práticas e valores podem modificar-se com a alteração do contexto onde se desenvolvem, incorporar a visão dos usuários na gestão das organizações públicas abre um caminho que favorece mudanças culturais nas relações entre estado e sociedade no setor saúde.

Observa-se, no entanto, que este tipo de estudo desvenda apenas parcialmente a dinâmica da prestação de cuidados nos serviços, devendo ser complementada com estudo de satisfação dos profissionais e avaliação dos serviços. Esta é uma limitação deste estudo que se ateve à visão dos usuários sobre o atendimento, propiciando um panorama parcial da qualidade dos serviços pesquisados. Além disso, dado o seu desenho, este estudo não permite generalizar seus achados para além das unidades pesquisadas. Importante ressaltar também que, embora a equipe de pesquisa tenha tido total independência para realizar a pesquisa, o fato de a equipe ser da Fiocruz avaliando unidades da Fiocruz pode ter introduzido um viés na pesquisa.

A disseminação de estudos sobre a satisfação de usuários constitui sem dúvida, um importante aspecto na avaliação dos serviços de saúde e dão visibilidade política aos usuários, um ator tradicionalmente pouco considerado na discussão das políticas no Brasil até recentemente.

\section{Colaboradores}

E Hollanda, SAV Siqueira, GRB Andrade e A Molinaro participaram igualmente de todas as etapas de elaboração do artigo. J Vaitsman participou como consultora do trabalho e de sua revisão final.

\section{Agradecimentos}

Os autores agradecem a colaboração do Centro de Saúde Escola Germano Sinval Faria

(CSEGSF), do Instituto de Pesquisas Evandro Chagas (IPEC) e do Instituto Fernandes Figueiras (IFF) pela receptividade e apoio a todas as fases do trabalho que permitiu a elaboração deste artigo. 


\section{Referências}

1. Andrade GRB, Vaitsman J, Farias LO. Metodologia de elaboração do Índice de Responsividade do Serviço (IRS). Cad Saude Publica 2010; 26(3):523-534.

2. Williams B. Patient satisfaction: a valid concept? Soc Sci Med 1994; 38(4):509-516.

3. Donabedian A. La calidad de la atención médica definición y métodos de evaluación. México: Prensa Médica Mexicana; 1984.

4. Esperidião MA, Trad LAB. Avaliação de satisfação de usuários: considerações teórico-conceituais. Cad. Saude Publica 2006; 22(6):1267-1276.

5. Kotaka F, Pacheco ML, Higaki Y. Avaliação pelos usuários dos hospitais participantes do programa de qualidade hospitalar do Estado de São Paulo, Brasil. Rev Saude Publica 1997; 31(2):171-177.

6. Shaw CD. Introducing quality assurance. London (UK): King's Fund Centre; 1986. (Project paper, n. 64).

7. Vuori H. Patient Satisfaction - an attribute or indicator of the quality of care? Qual Rev Bull. 1987; 13(3):106-108.

8. Esperidião MA, Trad LAB. Avaliação de satisfação de usuários. Cien Saude Colet 2005; 10(Supl.):S303S312.

9. Linder-Pelz S. Toward a theory of patient satisfaction. Soc Sci Med. 1982; 16(5):577-582.

10. Ramírez-Sánches TJ, Nájera-Aguilar P, NigendaLópes G. Percepción de la calidad de la atención de los servicios de salud en México: perspectiva de los usuarios. Sal Publ Mex. 1998; 40(1):3-12.

11. De Silva A. A framework for measuring responsiveness. Geneva: World Health Organization; 2000. (GPE Discussion Paper Series, n. 32)

12. Sitzia J, Wood N. Patient satisfaction: a review of issues and concepts. Soc Sci Med. 1997; 45(12):1829. 1843.

13. Paiva SMA, Gomes ELR. Assistência hospitalar: avaliação da satisfação dos usuários durante seu período de internação. Rev. Latino Am Enfermagem 2007; 15(5):973-979.

14. Brasil. Ministério da Saúde (MS). HumanizaSUS: política nacional de humanização. Ministério da Saúde, Secretaria-Executiva. Núcleo Técnico da Política Nacional de Humanização. Brasília: MS; 2003.

15. Pascoe GC. Patient satisfaction in primary health care: a literature review and analysis. Eval Progr Plann 1983; 6(3-4):185-210.

16. Bernhart $\mathrm{MH}$, Wiadnyana IGP, Wihardjo $\mathrm{H}$, Pohan I. Patient satisfaction in developing countries. Soc Sci Med. 1999; 48(8):9899-9996.

17. Baron-Epel O, Dushenat M, Friedman N. Evaluation of the consumer model: relationship between patient's expectations, perception and satisfaction with care. Int J Qual Health Care 2001; 13(4):317-323.

18. Gouveia GC, Souza WV, Luna CF, Souza-Júnior PRB, Szwarcwald CL. Satisfação dos usuários do sistema de saúde brasileiro: fatores associados e diferenças regionais. Rev Bras Epidemiol 2009; 12(3): 281-296.
19. Gouveia GC, Souza WV, Luna CF, Souza-Junio PRB, Szwarcwald CL. Health care user's satisfaction in Brazil, 2003. Cad. Saude Publica 2005; 21(Supl.): S109-S118.

20. World Health Organization (WHO). Health systems: improving performance. Geneva: The World Health Report; 2000.

21. Darby C, Valente N, Murray CJL, Amala S. World Health Organization (WHO): Strategy on Measuring Responsiveness. Geneva: WHO; 2000. (GPE Discussion Paper Series, n. 23)

22. Vaitsman J, Andrade GRB. Satisfação e responsividade: formas de medir a qualidade e a humanização da assistência à saúde. Cien Saude Colet. 2005; 10(3):599-613.

23. Gentil RM, Leal SMR, Scarpi MJ. Avaliação da resolutividade e da satisfação da clientela de um serviço de referência secundária em oftalmologia da Universidade Federal de São Paulo - UNIFESP. Arq Bras Oftalmol. 2003; 66(2):159-165.

24. Hollanda E, Siqueira SV, Andrade GRB, Pinto LF, Molinaro A, Sette ME, Vaitsman J. Satisfação dos usuários em três unidades de atenção à saúde da Fundação Oswaldo Cruz: Relatório final. Rio de Janeiro: Escola Nacional de Saúde Pública, Fundação Oswaldo Cruz; 2008

25. Conselho Nacional de Secretários Estaduais de Saúde (CONASS). Saúde na opinião dos brasileiros: um estudo prospectivo. Brasília: Editora do Ministério da Saúde; 2003.

26. Trad LAB, Bastos ACS. O impacto sócio-cultural do Programa de saúde da Família (PSF): uma proposta de avaliação. Cad Saude Publica 1988; 14(2):429435.

27. Muller EV, Greco M. Avaliação da satisfação dos usuários com os serviços do consórcio intermunicipal de saúde do noroeste do Paraná. Cien Saude Colet. 2010; 15(3):925-930.

28. Szwarcwald CL, Viacava F, Vasconcellos MTL, Lea MC, Azevedo LO, Queiroz RSB, Carvalho MF, Sou za Júnior PRB, Gama SGN, Fonseca MG. Pesquisa Mundial de Saúde - 2003: O Brasil em números. Radis 2004; 1(23):14-33.

29. Valentine N, Darby C, Bonsel GJ. Which aspects of non-clinical quality care are most important? Results from WHO's general population surveys of "health systems responsiveness" in 41 countries. Soc Sci Med. 2008; 66(9):1939-1950.

Artigo apresentado em 14/07/2011

Aprovado em 20/07/201

Versão final apresentada em 04/10/2011 